\title{
O impacto do Método Máe Canguru no processo de aprendizagem de prematuros de baixo peso: Revisão da literatura
}

\author{
Mariana de Paiva Franco ${ }^{\mathrm{a}}$, Cristiane Paiva Alves ${ }^{\mathrm{b}}$ \\ ${ }^{a}$ Faculdade de Medicina da Universidade de São Paulo - FMUSP, São Paulo, SP, Brasil \\ ${ }^{b}$ Departamento de Fisioterapia e Terapia Ocupacional, Universidade Estadual Paulista - UNESP, Marília, SP, Brasil
}

\begin{abstract}
Resumo: Os avanços da tecnologia e dos estudos científicos nas Unidades de Terapia Intensiva Neonatal (UTIN) contribuíram significativamente para a diminuição da mortalidade e da morbidade de recém-nascidos (RN) de risco, porém, eles continuam mais propensos a apresentar comprometimento neurológico e/ou retardo no desenvolvimento neuropsicomotor, com alterações neurológicas e sensoriais. Diante disso, surgiram propostas de programas de intervenção neonatal com o objetivo de resguardar o bebê e oferecer estímulos adequados para minimizar os efeitos das intervenções, com medidas protetoras, entre elas o Método Mãe Canguru (MMC). Face à relevância do tema descrito, esta pesquisa teve como objetivo realizar um levantamento bibliográfico de artigos nacionais e internacionais publicadas nos últimos anos (2000 a 2011) que descrevem de que maneira o MMC pode ou não agir como fator protetivo ao desenvolvimento da escrita em prematuros. Para a busca textual foi eleita a Biblioteca Virtual em Saúde - BVS, por se tratar de um site que abrange publicações mundiais, permitindo acesso a artigos da Ciências da Saúde, em geral tendo LILACS, IBECS, MEDLINE, Biblioteca Cochrane e SciELO como bases de dados. Os resultados encontrados nos artigos revelaram que os RN que participaram do MMC apresentaram melhoras no desenvolvimento e que fatores como a prematuridade, baixo peso ao nascer e distúrbios de aprendizagem estão fortemente correlacionados com deficiências motoras e alterações no desenvolvimento psicomotor. Concluiu-se que não foram encontrados artigos que descrevem o MMC como fator protetivo ao aparecimento da disgrafia. Sendo assim, acredita-se ser de grande importância a condução de futuros estudos sobre esses temas.
\end{abstract}

Palavras-chave: Revisão Acadêmica, Recém-Nascido Prematuro, Método Mãe Canguru, Disgrafia.

\section{The impact of the method Kangaroo Mother Care in the learning process of low-birth-weight preterm infants: A literature review}

\begin{abstract}
Technology advances and scientific studies in Neonatal Intensive Care Units (NICU) have contributed significantly to reduce mortality and morbidity of at-risk newborns (NB). However, they are more likely to present neurological and/or developmental psychomotor delay with neurological and sensory alterations. Therefore, proposals for neonatal intervention were developed with the aim of protecting the baby and offering appropriate incentives to minimize the effects of hospital intervention. To this end, programs of protective measures such as the Kangaroo Mother Care (KMC) were developed. Given the relevance of the issue described, this systematic review critically appraises articles from the national and international literature, published in recent years (from 2000 to 2011), that describe whether the KMC can be a protective factor for the development of writing in premature infants. The textual search was conducted using the Virtual Health Library (VHL), a website that covers publications worldwide, allowing access to articles from health science, including LILACS, IBECS, MEDLINE, Cochrane Library and SciELO, as database. The findings revealed that infants who participated in the KMC program showed improvements in their
\end{abstract}

Autor para correspondência: Mariana de Paiva Franco, Departamento de Fisioterapia, Fonoaudiologia e Terapia Ocupacional, Faculdade de Medicina, Universidade de São Paulo, Rua Cipotânea, 51, Cidade Universitária, Butantã, CEP 05360-160, São Paulo, SP, Brasil, e-mail: to.marianafranco@gmail.com

Recebido em 17/8/2011; Revisão em 18/5/2012; Aceito em 21/6/2012. 
development and that factors such as low-birth-weight prematurity and learning disorders have close relationship with the onset of motor impairments and changes in psychomotor development. The findings showed no articles describing the $\mathrm{KMC}$ as a protective factor for the incidence of dysgraphia. Thus, we emphasize the importance of conducting further studies on these topics.

Keywords: Bibliographic Review, Prematurity, Kangaroo Mother Care, Dysgraphia.

\section{Introdução}

Os avanços da tecnologia e os estudos científicos nas Unidades de Terapia Intensiva Neonatal (UTIN) contribuíram significativamente para a diminuição da mortalidade e da morbidade de recém-nascidos (RN) de risco, ou seja, aqueles prematuros nascidos com baixo peso e idade gestacional reduzida. Em contrapartida, esses avanços aumentaram a probabilidade do aparecimento de comprometimentos neurológicos e/ou retardo no desenvolvimento neuropsicomotor. As principias sequelas observadas são os distúrbios de aprendizagem e de comportamento e a paralisia cerebral (FAN, 2008).

Para Tronchin e Tsunechiro (2007), dentre os fatores que determinam o prognóstico e a qualidade de vida das crianças nascidas prematuramente estâo o grau de imaturidade fisiológica e anatômica dos sistemas, as condições ao nascer, as intercorrências clínicas e/ou de acordo com tratamento intensivo aos quais são submetidos. Ainda, a mesma fonte afirma que a criança nascida prematura também é susceptível a uma ampla variedade de problemas no neurodesenvolvimento, descritos como novas morbidades do recém-nascido prematuro de muito baixo peso, que abrangem desorganização de comportamento, falta de atenção, dificuldades nas áreas de linguagem, aprendizagem, integração visomotora, problemas sensoriais e perceptivos que podem se manifestar na primeira infância ou por ocasião do ingresso escolar (p. 80).

São classificadas como Recém-Nascidas Pré Termo (RNPT) crianças nascidas antes de serem completadas 37 semanas de gestação e RecémNascidas de Baixo Peso (RNBP) aquelas com peso de nascimento inferior ou igual a $2.500 \mathrm{~g}$, sendo subdivididas em recém-nascidas de muito baixo peso (peso de nascimento inferior a $1.500 \mathrm{~g}$ ) e recémnascidas de extremo baixo peso (peso de nascimento inferior a $1.000 \mathrm{~g}$ ), segundo a Organizaçáo Mundial da Saúde (WORLD..., 1977).

Venâncio e Almeida (2004) relatam que o Recém-Nascido Pré-Termo (RNPT) nasce em um período de maturação cerebral e de desenvolvimento do aparelho psíquico emocional. Sendo assim, o processo fisiológico de maturação é interrompido e o meio intrauterino que proporcionava experiências sensoriais e motoras é deixado, havendo um impacto considerável no seu organismo.

Estudos mostraram que se o meio no qual os prematuros sâo cuidados for inadequado, pode haver interferência negativa no seu desenvolvimento, acarretando alteraçóes no desenvolvimento global. Diante isso, surgiram propostas de programas de intervençáo neonatal com o objetivo de resguardar o bebê e oferecer estímulos adequados para minimizar os efeitos das intervençóes. Foram desenvolvidos programas que adotaram medidas protetoras, como a redução da luminosidade e do ruído do ambiente, adequação postural na incubadora para proporcionar a auto-organização e o autoconsolo, procedimentos menos dolorosos e menos invasivos e períodos de sono e descanso. A união dessas medidas com a possibilidade da presença dos pais na UTI neonatal e os cuidados e contato pele a pele trouxeram ganhos positivos para esses prematuros (VENÂNCIO; ALMEIDA, 2004).

O cuidado pele a pele foi denominado Método Mãe Canguru (MMC), definido pela Organização Mundial de Saúde (OMS) como o cuidado do Recém-Nascido de Baixo Peso (RNBP) que, após estabilização inicial, é mantido de forma contínua e prolongada em contato pele a pele. As crianças nascidas pré-termo e/ou com baixo peso ao nascer e com quadro estável apresentam perfil para indicação ao Método Mãe Canguru, pois necessitam de proteção térmica, alimentação adequada, observação frequente e proteção contra infecçôes. (CARDOSO et al., 2006).

O MMC, também conhecido como Cuidado Mãe Canguru ou Contato Pele a Pele, foi idealizado e implantado por Edgar Rey Sanabria e Hector Martinez em 1979, no Instituto Materno-Infantil de Bogotá, Colômbia, e tem sido proposto como uma alternativa ao cuidado neonatal convencional para bebês de baixo peso ao nascer (BPN). No Brasil, os primeiros serviços que aplicaram o MMC foram o Hospital Guilherme Álvaro, em Santos, SP, em 
1992, e o Instituto Materno-Infantil de Pernambuco (IMIP), em 1993 (VENÂNCIO; ALMEIDA, 2004).

O Ministério da Saúde do Brasil, na Portaria n. 693/GM, de 5 de julho de 2000, em seu artigo primeiro aprova a norma de orientação para a implantação do Método Canguru, destinado a promover a atençáo humanizada ao recém-nascido de baixo peso, definindo diretrizes para sua implantação nas unidades do Sistema Único de Saúde (SUS). Essa norma do Ministério da Saúde é dividida em três etapas de aplicação do método, sendo elas: 1) início nas unidades neonatais (unidades de terapia intensiva neonatal - UTIN e unidades de cuidados intermediários; 2) passando para as unidades canguru (ou alojamento conjunto canguru) até a alta hospitalar; 3) ambulatórios de seguimento (canguru domiciliar). Identificou-se na proposta brasileira cinco pilares, (1) cuidados individualizados, centrados nos pais (intervenção centrada na família); (2) contato pele a pele precoce (estimulação adequada e prazerosa, com integração sensorial); (3) controle ambiental de luz e som (para evitar estimulação aversiva e inadequada); (4) adequaçáo postural (prevenção de futuras distonias nos RN prematuros); (5) amamentação (favorecendo vínculo e prevenção de doenças no primeiro ano de vida) (BRASIL, 2000).

Cardoso et al. (2006) afirmam que as evidências científicas indicam que o Método Mãe Canguru traz benefícios à saúde do Recém-Nascido Baixo Peso (RNBP), reduzindo o custo e o tempo de internação hospitalar, humanizando a assistência, melhorando o vínculo mãe-filho e aumentando a adesão ao aleitamento materno exclusivo. Ressaltam que tanto no Brasil como nos países desenvolvidos o Método Mãe Canguru é uma proposta para uma parcela dos Recém-Nascidos Pré-Termo (RNPT) e não uma substituição da tecnologia utilizada nas UTIs neonatais.

As condiçóes associadas a fatores negativos ou indesejáveis, que comprometem a saúde, o bem-estar ou competência social são considerados fatores de risco, que podem determinar anormalidades no desenvolvimento neuro-sensório-motor (FORMIGA; PEDRAZZANI; TUDELLA, 2010).

Os recém-nascidos pré-termo e de baixo peso são considerados populaçấo de risco e dependem de atenção e cuidados especiais desde o nascimento, pois estáo suscetíveis a doenças que podem ocasionar alteraçôes em seu desenvolvimento. Devido a complicaçóes pré e perinatais, a maioria desenvolve precocemente ou tardiamente distúrbios na primeira infância ou na idade escolar, como dificuldades de aprendizagem ede linguagem, distúrbios de comportamento e da integração visomotora (AMARAL, TABAQUIM; LAMÔNICA, 2005).

Para Pesce et al. (2004), fatores positivos ou negativos ocorridos na infância podem manifestar seus impactos em longo prazo, havendo a possibilidade de experiências ocorridas nos primeiros anos de vida alterarem o curso do desenvolvimento ou influenciarem a forma como o indivíduo irá lidar com eventos estressores em fases posteriores, como na juventude e vida adulta.

Só é possível haver fatores de proteção se houver eventos estressores, pois sua função é alterar a resposta do indivíduo frente a uma dificuldade, complementam Formiga, Pedrazzani e Tudella (2010).

Os processos de proteção possuem quatro principais funçóes, sendo elas: (1) reduzir o impacto dos riscos, fato que altera a exposição da pessoa à situação adversa; (2) reduzir as reaçôes negativas em cadeia que se seguem à exposição do indivíduo à situação de risco; (3) estabelecer e manter a autoestima e autoeficácia, através do estabelecimento de relaçóes de apego seguras e do cumprimento de tarefas com sucesso; (4) criar oportunidades para reverter os efeitos do estresse (RUTTER, 1987 apud PESCE et al., 2004).

Formiga, Pedrazzani e Tudella (2010) ressaltam que esses fatores podem ser recursos do próprio indivíduo ou do ambiente em que estão inseridos, os quais podem neutralizar os riscos, adversidades e vulnerabilidades ao seu desenvolvimento.

Os fatores de proteção são necessários para o restabelecimento do equilíbrio perdido frente às situações geradoras de desequilíbrio. Há três tipos de fatores de proteção para a criança/adolescente: (1) fatores individuais: autoestima positiva, autocontrole, autonomia, características de temperamento afetuoso e flexível; (2) fatores familiares: coesão, estabilidade, respeito mútuo, apoio/suporte; (3) fatores relacionados ao apoio do meio ambiente: bom relacionamento com amigos, professores ou pessoas significativas que assumam papel de referência segura para a criança e a façam sentir-se querida e amada (EMERY; FOREHAND, 1996 apud PESCE et al., 2004).

Segundo Fan (2008), há uma estimativa de que as crianças prematuras apresentem até $50 \%$ a mais a probabilidade de necessitarem de educação especial quando comparadas às crianças nascidas a termo, sendo a dificuldade de aprendizado uma das principais causas de tal fato, mesmo que sem maiores deterioraçóes no neurodesenvolvimento.

Os transtornos de aprendizagem, segundo o DSM-IV (ASSOCIAÇÃO..., 1995), caracterizam-se por um funcionamento acadêmico abaixo do 
esperado, considerando a idade cronológica, medidas de inteligência e educaçáo apropriadas à idade, e são divididos em transtorno da leitura, transtorno da matemática, transtorno da expressão escrita e transtorno da aprendizagem sem outra especificação. Os processos de aprendizagem da leitura e da escrita envolvem habilidades cognitivas complexas, a linguagem escrita abrange a habilidade motora, a sensório-perceptual e as socioemocionais (FAN, 2008).

As crianças que apresentam dificuldades no ato motor da escrita, no que diz respeito ao traçado das letras e à disposição dos conjuntos gráficos no espaço utilizado, são consideradas disgráficas. Apresentam, portanto, dificuldades motoras e espaciais (CINEL, 2003).

Rodrigues, Castro e Ciasca (2008) descrevem a disgrafia como uma dificuldade de produzir uma escrita aceita culturalmente, apesar de os indivíduos possuírem um nível intelectual adequado e serem submetidos ao mesmo processo de formação acadêmica com prática da escrita. A disgrafia pode ser classificada como secundária a uma lesão - disgrafia adquirida (perda de habilidades anteriormente adquiridas) ou como uma disfunção do Sistema Nervoso Central (SNC): disgrafia do desenvolvimento (desenvolvimento anormal da habilidade de escrever), disgrafia funcional (utilizada para aqueles indivíduos com capacidade intelectual normal ou acima da média, sem déficits sensoriais e lesôes neurológicas) e disgrafia orgânica (onde há déficits intelectuais, sensoriais ou lesão do SNC).

Amaral, Tabaquim e Lamônica (2005) avaliam que atrasos no desenvolvimento motor têm consequências importantes, que alteram as interaçôes ambientais e o desempenho de habilidades da vida diária, instalando-se uma sequência desorganizada, dependendo da etiologia específica que levou a esse atraso. O desenvolvimento motor é fundamental para a criança, pois com ele elas desenvolvem habilidades de relação e exploração do ambiente, através da linguagem, manipulação dos objetos e do próprio corpo e da repetição de açóes.

Venâncio e Almeida (2004) complementam dizendo que as crianças prematuras que receberam o cuidado pele a pele apresentaram melhores índices em testes de motricidade e melhor desenvolvimento mental.

Face à relevância do tema descrito, esta pesquisa teve como objetivo realizar um levantamento bibliográfico de artigos nacionais e internacionais publicadas nos últimos anos (2000 a 2011) que descrevem de que maneira o MMC pode ou não agir como fator protetivo ao desenvolvimento da escrita em prematuros.

\section{Material e método}

\subsection{Levantamento bibliográfico}

Este estudo consistiu em uma pesquisa bibliográfica sobre pesquisas que avaliavam crianças, adolescentes ou adultos nascidos prematuros que foram submetidos ao MMC, analisando sua açáo protetiva, a curto, médio e longo prazos. Além das pesquisas que avaliaram a relaçáo entre prematuridade e/ou baixo peso ao nascer com transtornos de aprendizagem.

Para tal levantamento, foi eleita a Biblioteca Virtual em Saúde - BVS (http://regional.bvsalud. org/php/decsws.php), por se tratar de um site que abrange publicaçôes mundiais, permitindo acesso à:

- Literatura científica e técnica;

- Artigos da ciências da saúde em geral (LILACS, IBECS, MEDLINE, Biblioteca Cochrane, SciELO);

- Portal de evidências;

- Avaliaçôes econômicas em saúde;

- Avaliaçóes de tecnologias em saúde;

- Diretrizes para a prática clínica;

- Áreas especializadas;

- Organismos internacionais (PAHO, WHOLIS);

- LIS - Localizador de Informação em Saúde;

- DeCS - Terminologia em saúde;

- Documentos (SCAD - serviço de cópia de documentos, catálogo de revistas científicas);

- 1.807.251 artigos com textos completos.

Após a realização da busca dos descritores na BVS, não foi encontrada nenhuma publicação relacionando o Método Mãe Canguru com transtornos de aprendizagem. Descritores utilizados:

- kangaroo mother care > learning disorder;

- kangaroo mother care > learning difficulties;

- kangaroo mother care > fine motor development;

- kangaroo mother care > disgraphy; igualmente ausentes para os termos em português.

Optou-se, portanto para a divisão em duas etapas. Essa divisão foi necessária pois os descritores presentes nos artigos relacionados, no Tabela 1, 
correlacionavam-se de diferentes formas. Dessa forma, chegou-se aos descritores do Tabela 2.

\subsection{Critérios de seleção}

Os critérios de inclusão para as partes I e II foram: artigos com textos completos cujo foco da pesquisa fosse procedimentos ou avaliações com recém-nascidos, crianças, adolescentes ou adultos nascidos prematuros e/ou com baixo peso ao nascer como sujeitos diretos da pesquisa, publicadas entre os anos 2000 a 2011.

Foram excluídos os textos incompletos, teses, artigos de revisão, artigos teóricos, guias, manuais, avaliaçóes institucionais e entrevistas com pais, comunidade e profissionais da saúde.

O resultado final da triagem para a análise foram 18 artigos da parte I e 11 artigos da parte II, resultando em 29 artigos.

\section{Resultados}

\subsection{Caracterização das fontes de análise}

Os artigos selecionados foram publicados entre 2001 e 2009, conforme Figura 1.

Dentro da seleção realizada, no ano de 2008 houve o maior número de publicações.

Para estabelecer a relaçáo entre número de artigos, periódicos em que foram publicados e base de dados foi elaborado o Tabela 3.

\subsection{Exposição dos resultados}

Os dados dos 29 artigos foram reunidos, sistematizados e apresentados em forma de tabela (Tabela 4), onde podem ser observadas algumas

Tabela 1. Parte I - Número de artigos completos, número de artigos já inclusos em outras seleções e número de resumos para triagem.

\begin{tabular}{lccc}
\hline \multicolumn{1}{c}{ Descritores } & $\begin{array}{c}\text { Artigos com } \\
\text { texto completo }\end{array}$ & $\begin{array}{c}\text { Artigos já inclusos } \\
\text { nas seleções } \\
\text { anteriores }\end{array}$ & $\begin{array}{c}\text { Resumos para } \\
\text { triagem }\end{array}$ \\
\hline kangaroo mother care & 60 & 0 & 60 \\
cuidado mãe canguru & 16 & 15 & 1 \\
método mãe canguru & 20 & 16 & 4 \\
kangaroo mother method & 38 & 35 & 3 \\
kangaroo mother program & 13 & 12 & 1 \\
programa mãe canguru & 10 & 10 & 0 \\
preterm infants $>$ kangaroo mother care & 5 & 5 & 0 \\
premature low birth weight $>$ kangaroo mother care & 8 & 8 & 0 \\
Total & - & - & 69 \\
\hline
\end{tabular}

Tabela 2. Parte II - Número de artigos completos, número de artigos já inclusos em outras seleções e número de resumos para triagem.

\begin{tabular}{lccc}
\hline \multicolumn{1}{c}{ Descritores } & $\begin{array}{c}\text { Artigos } \\
\text { com texto } \\
\text { completo }\end{array}$ & $\begin{array}{c}\text { Artigos já inclusos } \\
\text { nas seleçóes } \\
\text { anteriores }\end{array}$ & $\begin{array}{c}\text { Resumos } \\
\text { para } \\
\text { triagem }\end{array}$ \\
\hline premature low birth weight > learning disorder & 6 & 0 & 6 \\
prematuro $>$ transtornos de aprendizagem & 8 & 4 & 4 \\
prematuro com baixo peso > transtornos de aprendizagem & 1 & 1 & 0 \\
premature children > fine motor development & 5 & 1 & 4 \\
preterm > learning difficulties & 4 & 0 & 4 \\
Total & - & - & 18 \\
\hline
\end{tabular}


Tabela 3. Número de artigos publicados e respectivos periódicos e bases de dados.

\begin{tabular}{lcc}
\hline \multicolumn{1}{c}{ Periódico } & Base de dados & $\begin{array}{c}\text { Número de } \\
\text { artigos }\end{array}$ \\
\hline BMC Pediatrics & MEDLINE /Pubmed & 1 \\
Pain Management Nursing & MEDLINE /Pubmed & 1 \\
Jornal de Pediatria (Rio Janeiro) & LILACS & 4 \\
Journal of Obstetric, Gynecologic, and Neonatal Nursing & MEDLINE /Pubmed & 1 \\
Revista Brasileira de Saúde Materno Infantil & LILACS & 2 \\
Revista Brasileira de Fisioterapia & LILACS & 1 \\
Acta Paulista de Enfermagem & LILACS & 1 \\
AACN Clinical Issues & MEDLINE /Pubmed & 1 \\
Revista Eletrônica de Enfermagem & LILACS & 1 \\
Revista Cubana de Pediatria & LILACS & 3 \\
Pró-Fono Revista de Atualização Científica & LILACS & 1 \\
Texto \& Contexto Enfermagem & LILACS & 1 \\
Colombia Médica & LILACS & 1 \\
Archives of Disease in Childhood - Fetal and Neonatal Edition & MEDLINE /Pubmed & 5 \\
Revista del Hospital Materno Infantil Ramón Sarda & LILACS & 1 \\
Pediatrics & MEDLINE /Pubmed & 2 \\
BMC Medicine & MEDLINE /Pubmed & 1 \\
Revista CEFAC & LILACS & 1 \\
Total & & $\mathbf{2 9}$ \\
\hline
\end{tabular}

NÚMERO DE ARTIGOS POR ANO

= NÚMERO DE ARTIGOS POR ANO

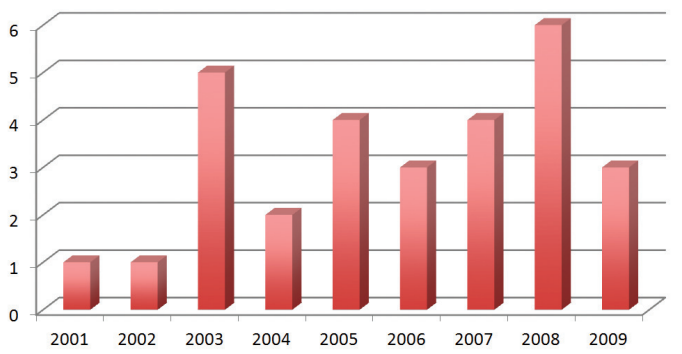

Figura 1. Número de artigos por ano.

características dos artigos analisados como: autores, ano de publicação, objetivos, participantes e idioma.

\section{Discussão}

Como pode ser observado, foram publicados em inglês (13), em português (12) e em espanhol (4).

De acordo com o critério de inclusão, todas as 29 pesquisas foram realizadas com crianças, adolescentes e adultos nascidos prematuros. Entre elas, 14 avaliaram recém-nascidos, quatro avaliaram crianças de 4 a 34 meses, dois, crianças de 2 a 3 anos, quatro, crianças em idade pré-escolar $(5$ a 7 anos), cinco foram realizados com adolescentes (12 a 16 anos) e duas com jovens adultos (19 a 22 anos, sendo as pesquisas que avaliaram prematuros com maior idade). Vale ressaltar que em alguns artigos houve uma abrangência maior de faixa etária, não correspondendo o número de artigos com o descrito pelas faixas etárias.

Os instrumentos de coletas de dados foram variados, sendo qualitativos e quantitativos. O Teste de Inteligência de Wechsler para Crianças (Wechsler III) está presente em três artigos, a Escala de Bayley foi utilizada em dois artigos. O instrumento mais citado foi a Escala de Inteligência Wechsler para Crianças (WISC III) - que será descrita a seguir, para maior entendimento de sua utilização nos artigos analisados.

WISC III - Utilizada para a avaliação da inteligência de crianças com idade entre 6 e 16 anos, 11 meses e 30 dias. É composta por seis subtestes: Área verbal (informação, compreensão, semelhanças, vocabulário, aritmética e dígitos) e Área de execução (avalia principalmente aspectos maturativos cerebrais, formada por atividades de completar figuras, arranjos de figuras, cubos, armar objetos e códigos, procurar símbolos e labirintos (COSTA et al., 2004 apud FAN, 2008).

Os dados encontrados nos artigos selecionados revelaram que os RN que participaram do MMC apresentaram resultados significativamente positivos, como melhoria no aleitamento materno e no tempo de permanência na internação, temperatura corporal e saturação periférica de oxigênio aumentadas, 


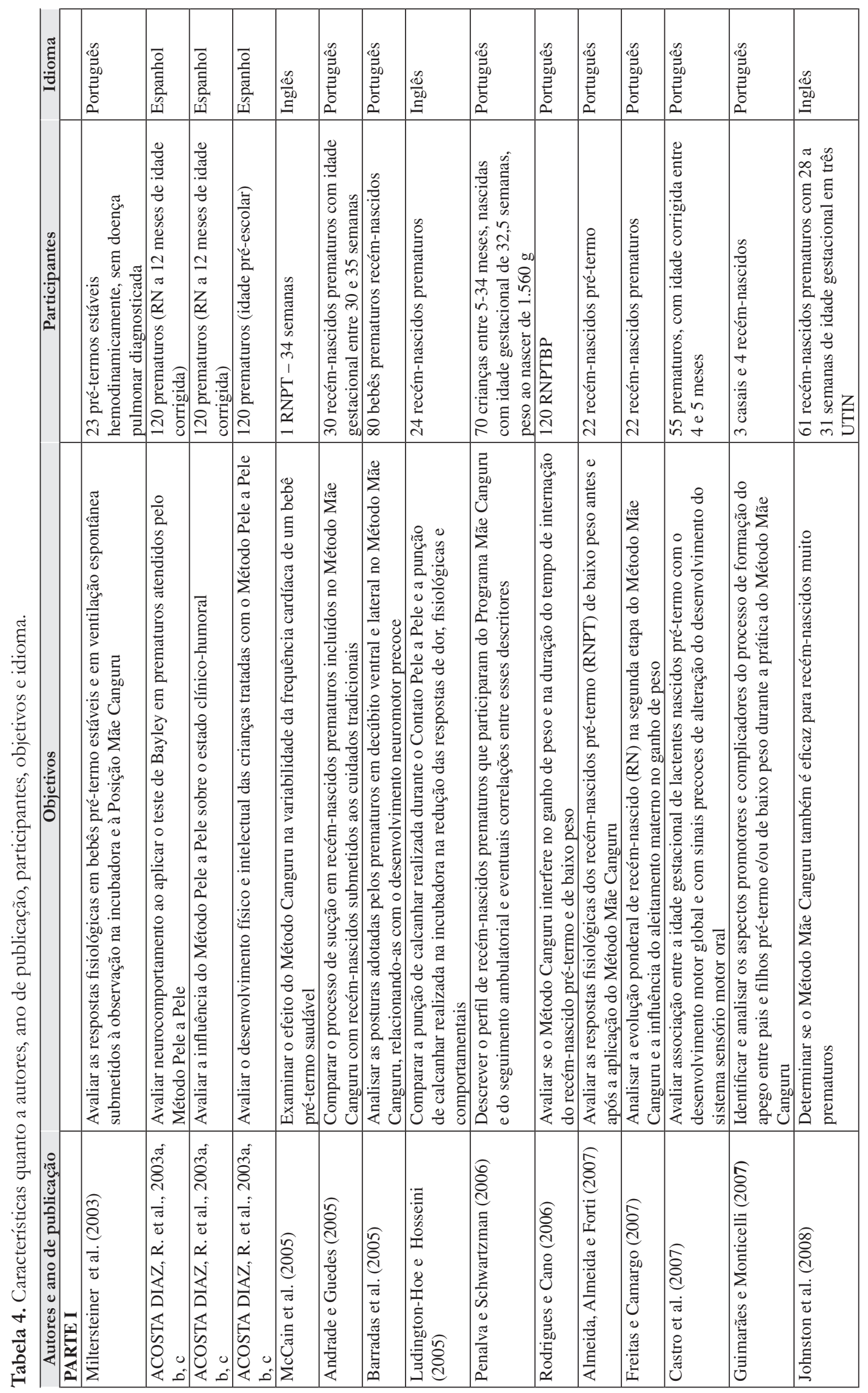




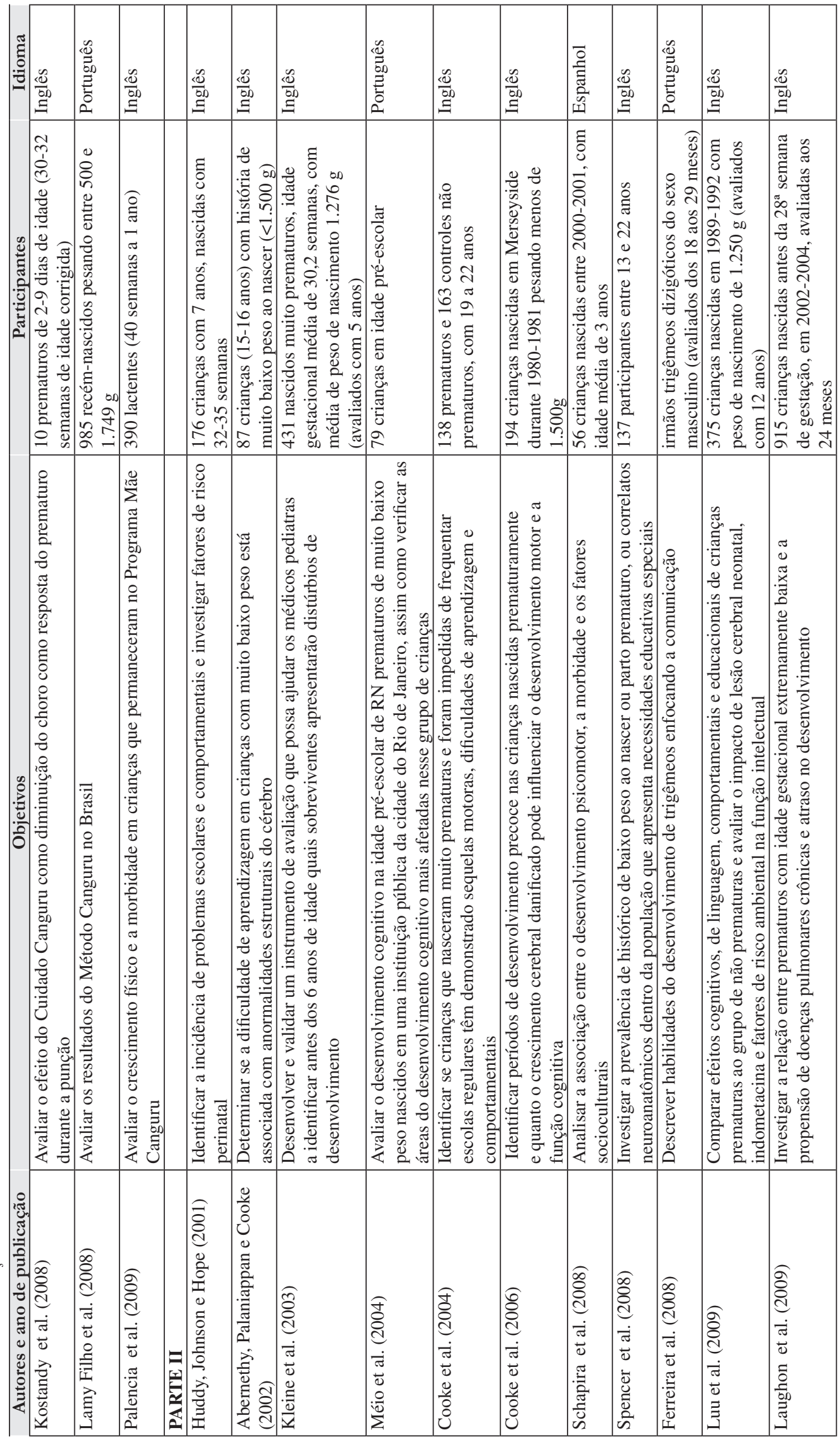


maior conforto respiratório, aumento das respostas fisiológicas, recuperação mais rápida em relação aos batimentos cardíacos, diminuição do choro e sinais de dor após estímulo doloroso, fatos que, segundo Johnston et al. (2008), parecem estar relacionados a mecanismos endógenos, produzidos pelo contato pele a pele, o que ocasiona a diminuição da dor, ajudando a manutenção da homeostase. Observaram-se também diminuição de reinternaçôes devidas a infecçôes, diminuição de stress, melhor comportamento neuromotor, assim como melhora no desenvolvimento sensório motor oral e global, formação e manutenção dos processos afetivos entre família e RN. Corroborando dados da pesquisa de Venâncio e Almeida (2004), mostraram que se o meio no qual os prematuros são cuidados for inadequado, pode haver interferência negativa no seu desenvolvimento, acarretando alteraçóes no desenvolvimento global. Pesce et al. (2004) também destacam que fatores positivos ou negativos ocorridos na infância podem manifestar seus impactos em longo prazo, havendo a possibilidade de experiências ocorridas nos primeiros anos de vida alterarem o curso do desenvolvimento ou influenciarem a forma como o indivíduo irá lidar com eventos estressores em fases posteriores, como na juventude e vida adulta.

Os estudos destacaram, ainda, a simplicidade e o custo reduzido do MMC, apresentando-o como ótima alternativa para países em desenvolvimento, pois há a efetividade na amamentação e diminuição do tempo de internaçáo, mostrando-se, também, uma boa estratégia para a manutenção e estímulo ao aleitamento materno (PENALVA; SCHWARTZMAN, 2006; LAMY FILHO et al., 2008). Assim como preconiza o Ministério da Saúde do Brasil na Portaria n. 693/GM, de 5 de julho de 2000, artigo primeiro, que aprova a Norma de Orientação para a Implantação do Método Canguru, destinado a promover a atenção humanizada ao recém-nascido de baixo peso, definindo diretrizes para sua implantação nas unidades do Sistema Único de Saúde (SUS) (BRASIL, 2000).

Uma minoria de artigos analisados relatou resultados pouco significativos em testes físicos e em ganho de peso. Salienta-se que estudos de curto, médio e longo prazos sáo importantes, assim como pesquisas para avaliar o desenvolvimento motor.

Já em relação à prematuridade, baixo peso e transtornos de aprendizagem, as pesquisas mostraram que esses fatores estão fortemente relacionados uns com os outros e podem predizer deficiências motoras, baixo QI, afetar o desenvolvimento neuropsicomotor, predizer déficits de atenção, dispraxia, que pode estar relacionada ao crescimento global do cérebro, e afetar o desenvolvimento de estruturas essenciais como o núcleo caudado e formaçóes do hipocampo (ABERNETHY; PALANIAPPAN; COOKE, 2002).

Assim como Tronchin e Tsunechiro (2007), que relatam que a criança nascida prematura também é susceptível a uma ampla variedade de problemas no neurodesenvolvimento, descritos como novas morbidades do recém-nascido prematuro de muito baixo peso, os quais abrangem desorganizaçáo de comportamento, falta de atenção, dificuldades nas áreas de linguagem, aprendizagem, integração visomotora, problemas sensoriais e perceptivos que podem se manifestar na primeira infância ou por ocasiáo do ingresso escolar (p. 80).

Na pesquisa de Huddy, Johnson e Hope (2001) afirma-se que até um terço das crianças nascidas entre 32 e 35 semanas de gestação podem ter problemas na escola. Dados que corroboram os de Venâncio e Almeida (2004), que relataram que o Recém-Nascido Pré-Termo (RNPT) nasce em um período de maturação cerebral e de desenvolvimento do aparelho psíquico emocional. Sendo assim, o processo fisiológico de maturação é interrompido, e o meio intrauterino que proporcionava experiências sensoriais e motoras é deixado, havendo um impacto considerável no seu organismo. Essas descobertas têm implicações para os serviços educacionais, portanto uma intervenção adequada e precoce é necessária para evitar ou reduzir problemas durante a escolaridade. Fatos que corroboram a pesquisa de Fan (2008, p. 24), que diz haver uma estimativa de que as crianças prematuras apresentem até $50 \%$ mais probabilidades de necessitarem de educação especial, quando comparadas a crianças nascidas a termo, sendo a dificuldade de aprendizado uma das principais causas para tal fato, mesmo que sem maiores deterioraçóes no neurodesenvolvimento.

Frente aos dados apresentados podemos sugerir que o MMC é um fator protetivo, visto que os processos de proteção possuem quatro principais funções: (1) reduzir o impacto dos riscos, fato que altera a exposição da pessoa à situação adversa; (2) reduzir as reaçóes negativas em cadeia, que seguem-se à exposição do indivíduo à situação de risco; (3) estabelecer e manter a autoestima e autoeficácia através de estabelecimento de relaçóes de apego seguras e do cumprimento de tarefas com sucesso; (4) criar oportunidades para reverter os efeitos do estresse. Os fatores de proteção são necessários para o restabelecimento do equilíbrio perdido frente às situaçóes geradoras de desequilíbrio (RUTTER, 1987 apud PESCE et al., 2004). Já a prematuridade se enquadra nos fatores de risco para 
o desenvolvimento do $\mathrm{RN}$, pois eles dependem de atenção e cuidados especiais desde o nascimento, estão suscetíveis a doenças que podem ocasionar alteraçôes em seu desenvolvimento. Devido a complicaçóes pré e perinatais, a maioria desenvolve precoce ou tardiamente distúrbios na primeira infância ou na idade escolar, como dificuldades de aprendizagem e de linguagem, distúrbios de comportamento e da integração viso motora (AMARAL; TABAQUIM; LAMÔNICA, 2005). As condiçôes associadas a fatores negativos ou indesejáveis, que comprometem a saúde, o bem-estar ou competência social são consideradas fatores de risco que podem determinar anormalidades no desenvolvimento neuro-sensóriomotor. Portanto, náo foram encontrados, na busca teórica, pesquisas que mostrem o MMC como fator protetivo no distúrbio de aprendizagem, assim como na disgrafia. O presente artigo buscou fundamentar teoricamente e analisar a relação entre os fatores risco e proteçáo, reafirmando a relevância de uma futura proposta de pesquisa, propondo um estudo aprofundado que investigue os benefícios do Método Mãe Canguru para o Recém-Nascido Pré-Termo (RNPT) e com Baixo Peso ao Nascer (BPN) no desenvolvimento da coordenação motora fina (escrita) na idade escolar. Amaral, Tabaquim e Lamônica (2005) avaliam que quando ocorre atraso no desenvolvimento motor há consequências importantes, que alteram as interaçôes ambientais e o desempenho de habilidades da vida diária, seguindo-se uma sequência desorganizada, dependente da etiologia específica que levou a esse atraso. O desenvolvimento motor é fundamental para a criança no desenvolvimento de habilidades de relação e na exploração do ambiente, através da linguagem, da manipulação de objetos e do próprio corpo e da repetição de açôes. Venâncio e Almeida (2004) complementam afirmando que as crianças prematuras que receberam o cuidado pele a pele apresentaram melhores índices em testes de motricidade e melhor desenvolvimento mental. Ainda, segundo Acosta Diaz (2003c), a idade pré-escolar é um período no qual são estruturadas as bases fundamentais do desenvolvimento da personalidade, regulando uma série de mecanismos fisiológicos que influenciam o desenvolvimento físico, o estado de saúde, o grau de adaptação ao meio e o desenvolvimento intelectual, que pode aparecer alterado no prematuro dessa idade. Com o MMC tais mecanismos podem ser modificados desde o nascimento, como uma forma de estimulação precoce (FORMIGA; PEDRAZZANI; TUDELLA, 2010).

\section{Considerações finais}

De acordo com o exposto, concluiu-se que náo existem artigos que relacionem diretamente o MMC como fator protetivo para o desenvolvimento da disgrafia. Portanto, enfatiza-se a importância da realizaçáo de estudos relacionados a esses temas que avaliem o impacto do MMC nos processos de aprendizagem. Especificamente, na sua relação com a disgrafia na população sobrevivente à prematuridade e ao baixo peso ao nascer.

\section{Referências}

ACOSTA DIAZ, R. et al. Método piel a piel: Evaluación del neurocomportamiento hasta el año de edad corregida. Revista Cubana de Pediatria, Ciudad de la Habana, v. 75, n. 3, 2003a. Disponível em: <http://scielo.sld.cu/scielo. php?script=sci_arttext\&pid=S00347531200300030000 2\&lng=es\&nrm=iso >. Acesso em: 05 jun. 2011.

ACOSTA DIAZ, R. et al. Método piel a piel: Evaluación clínica-humoral durante el primer año de edad corregida. Revista Cubana de Pediatria, Ciudad de la Habana, v. 75, n. 3, sept. 2003b. Disponível em: <http://scielo.sld.cu/ scielo.php?script=sci_arttext\&pid $=S 003475312003000$ 300004\&lng=es\&nrm=iso>. Acesso em: 05 jun. 2011.

ACOSTA DIAZ, R. et al. Método piel a piel: Repercusión sobre el desarrollo físico-intelectual a la edad preescolar. Revista Cubana de Pediatria, Ciudad de la Habana, v. 75, n. 3, sept. 2003c. Disponível em: <http://scielo.sld.cu/ scielo.php?script=sci_arttext\&pid=S003475312003000 300003\&lng=es\&nrm=iso >. Acesso em: 05 jun. 2011.

ABERNETHY, L. J.; PALANIAPPAN, M.; COOKE, R. W. Quantitative magnetic resonance imaging of the brain in survivors of very low birth weight. Archives of Disease in Childhood, London, v. 87, n. 4, p. 279-283, 2002. PMCid:PMC1763037. http://dx.doi.org/10.1136/ adc.87.4.279

ALMEIDA, C. M.; ALMEIDA, A. F. N.; FORTI, E. M. P. Efeitos do Método Mãe Canguru nos sinais vitais de recém-nascidos pré-termo de baixo peso. Revista Brasileira de Fisioterapia, São Carlos, v. 11, n. 1, p. 1-5, 2007. http:// dx.doi.org/10.1590/S1413-35552007000100002

ANDRADE, I. S. N.; GUEDES, Z. C. F. Sucção do recém-nascido prematuro: comparação do método mãe-canguru com os cuidados tradicionais. Revista Brasileira de Saúde Materno Infantil, Recife, v. 5, n. 1, p. 61-69, 2005.

AMARAL, A. C. T.; TABAQUim, M. L. M.; LAMÔNICA, D. A. C. Avaliação de habilidades de crianças com alteração no desenvolvimento. Relato de Pesquisa. Revista Brasileira de Educação Especial, Marília, v. 11, n. 2, p. 185-200, 2005.

ASSOCIAÇÃO AMERICANA DE PSIQUIATRIA APA. Manual diagnóstico e estatístico de transtornos mentais (DSM-IV). 4. ed. Porto Alegre: Artes Médicas; 1995. 
BARRADAS, J. et al. Relationship between positioning of premature infants in Kangaroo Mother Care and early neuromotor development. Jornal de Pediatria, Porto Alegre, v. 82, n. 6, p. 475-480, 2006. PMid:17171208. http://dx.doi.org/10.2223/JPED.1565

BRASIL. Ministério da Saúde. Portaria no 693/GM 05 de julho de 2000. Norma de orientação para a implantação do Método Canguru. Brasília: Ministério da Saúde, 2000. Disponível em: <http://www.portal.saude.gov.br/portal/ arquivos/pdf/Port.\%20693\%20MMC.pdf>. Acesso em: 24 maio 2011.

CARDOSO, A. C. A. et al. Método Mãe-Canguru: aspectos atuais. Pediatria, Sáo Paulo, v. 28, n. 2, p. 128-134, 2006.

CASTRO, A. G. et al. Desenvolvimento do sistema sensório motor oral e motor global em lactentes pré-termo. Pró-Fono, São Paulo, v. 19, n. 1, p. 29-38, 2007. http:// dx.doi.org/10.1590/S0104-56872007000100004

CINEL, N. C. B. Disgrafia: Prováveis causas dos distúrbios e estratégias para a correçáo da escrita. Revista do Professor, Belo Horizonte, v. 19, n. 74, p. 19-25, 2003.

COOKE, R. W. Are there critical periods for brain growth in children born preterm? Archives of Disease in Childhood: Fetal and Neonatal Edition, London, v. 91, n. 1, p. 17-20, 2006. PMid:16223756 PMCid:PMC2672640. http://dx.doi.org/10.1136/adc.2005.077438

COOKE, R. W. Health, lifestyle, and quality of life for young adults born very preterm. Archives of Disease in Childhood, London, v. 89, n. 3, p. 201-6, 2004. PMCid:PMC1719850. http://dx.doi.org/10.1136/ adc.2003.030197

FAN, R. G. Aprendizado e comportamento em crianças nascidas prematuras e com baixo peso em idade pré-escolar e em processo de alfabetização. 2008. 144 f. Dissertação (Mestrado em Medicina e Ciências da Saúde)-Pontifícia Universidade do Rio Grande do Sul, Porto Alegre, 2008.

FERREIRA, A. T. et al. Desempenho comunicativo em trigêmeos prematuros. Revista CEFAC, São Paulo, v. 10, n. 1, p. 15-21, 2008. http://dx.doi.org/10.1590/ S1516-18462008000100003

Freitas, J. O.; CAMARGO, C. L. Método Máe-Canguru: evolução ponderal de recém-nascidos. Acta Paulista de Enfermagem, São Paulo, v. 20, n. 1, p. 75-81, 2007. http://dx.doi.org/10.1590/ S0103-21002007000100013

FORMIGA, C. K. M. R.; PEDRAZZANI, E. S.; TUDELLA, E. Intervenção precoce com bebês de risco. São Paulo: Editora Atheneu, 2010.

GUIMARĀES, G. P.; MONTICELLI, M. A formação do apego pais/recém-nascido pré-termo e/ou de baixo peso no método mãe-canguru: uma contribuição da enfermagem. Texto \& Contexto de Enfermagem, Florianópolis, v. 16, n. 4, p. 626-35, 2007.

HUDDY, C. L.; JOHNSON, A.; HOPE, P. L. Educational and behavioral problems in babies of 32-35 weeks gestation. Archives of Disease in Childhood: fetal and neonatal edition, London, v. 85, n. 1, p. 23-28, 2001.
JOHNSTON, C. C. et al. Kangaroo mother care diminishes pain from heel lance in very preterm neonates: a crossover trial. BMC Pediatrics, London, v. 8, n. 13, p. 03-09, 2008.

KLEINE, M. J. et al. Development and evaluation of a follow up assessment of preterm infants at 5 years of age. Archives of Disease in Childhood, London, v. 88, n. 10, p. 870-875, 2003. PMCid:PMC1719302. http://dx.doi. org/10.1136/adc. 88.10 .870

KOSTANDY, R. R. et al. Kangaroo Care (skin contact) reduces crying response to pain in preterm neonates: pilot results. Pain Management Nursing, Philadelphia, v. 9, n. 2, p. 55-65, 2008. PMid:18513662 PMCid:PMC2647362. http://dx.doi.org/10.1016/j.pmn.2007.11.004

LAMY FILHO, F. et al. Avaliação dos resultados neonatais do método canguru no Brasil. Jornal de Pediatria, São Paulo, v. 84, n. 5, p. 428-435, 2008. http://dx.doi. org/10.1590/S0021-755720080006000

LAUGHON, M. et al. Chronic lung disease and developmental delay at 2 years of age in children born before 28 weeks' gestation. Pediatrics, Houston, v. 124, n. 2, p. 637-48, 2009. PMid:19620203 PMCid:PMC2799188. http://dx.doi.org/10.1542/peds.2008-2874

LUDINGTON-HOE, S. M.; HOSSEINI, R. Skin-to-skin contact (Kangaroo Care) analgesia for preterm infant heel stick. AACN Clinical Issues, Philadelphia, v. 16, n. 3, p. 373-387, 2005. http://dx.doi. org/10.1097/00044067-200507000-00010

LUU, T. M. et al. Lasting effects of preterm birth and neonatal brain hemorrhage at 12 years of age. Pediatrics, Houston, v. 123, n. 3, p. 1037-1044, 2009. PMid:19255037 PMCid:PMC2651566. http://dx.doi. org/10.1542/peds.2008-1162

McCAIN, G. C. et al. Heart rate variability responses of a preterm infant to kangaroo care. Journal of Obstetric, Gynecologic, \& Neonatal Nursing, Malden, v. 34, n. 6, p. 689-694, 2005. PMid:16282226 PMCid:PMC2133345. http://dx.doi.org/10.1177/0884217505281857

MÉIO, M. D. et al. Pre-school cognitive development of very low birth weight preterm children. Jornal de Pediatria, Sáo Paulo, v. 80, n. 6, p. 495-502, 2004. http://dx.doi. org/10.2223/JPED.1264

MILTERSTEINER, A. R. et al. Respostas fisiológicas da Posição Mãe-Canguru em bebês pré-termos, de baixo peso e ventilando espontaneamente. Revista Brasileira de Saúde Materno Infantil, Recife, v. 3, n. 4, p. 447-455, 2003. http://dx.doi.org/10.1590/S1519-38292003000400009 PALENCIA, D. et al. Kangaroo mother program: physical growth and morbidity in a cohort of children, followed from 40 weeks of postconceptional age until first year. Colombia Médica, Cali, v. 40, n. 3, p. 292-299, 2009.

PENALVA, O.; SCHWARTZMAN, J. Descriptive study of the clinical and nutritional profile and follow-up of premature babies in a Kangaroo Mother Care Program. Jornal de Pediatria, São Paulo, v. 82, n. 1, p. 33-39, 2006. PMid:16532145. http://dx.doi.org/10.2223/JPED.1434 
PESCE, R. P. et al. Risco e proteção: em busca de um equilíbrio promotor de resiliência. Psicologia: Teoria e Pesquisa, Brasília, v. 20, n. 2, p. 135-143, 2004. http:// dx.doi.org/10.1590/S0102-37722004000200006

RODRIGUES, S. D.; CASTRO, M. J. M. G; CIASCA, S. M. Relação entre indícios de disgrafia funcional e desempenho acadêmico. Revista CEFAC, São Paulo, v. 11, n. 2, p. 221-227, 2008. http://dx.doi.org/10.1590/ S1516-18462008005000005

RODRIGUES, M. A. G.; CANO, M. A. T. Estudo do ganho de peso e duração da internaçáo do recém-nascido pré-termo de baixo peso com a utilização do método canguru. Revista Eletrônica de Enfermagem, Goiânia, v. 8, n. 2, p. 185-191, 2006.

SCHAPIRA, I. et al. Desarrollo alejado de niños nacidos prematuros. Revista del Hospital Materno Infantil Ramón Sardá, Buenos Aires, v. 27, n. 4, p. 155-158, 2008.

SPENCER, M. D. et al. Low birthweight and preterm birth in young people with special educational needs: a magnetic resonance imaging analysis. BMC Medicine, London, v. 6, n. 1, p 1-11, 2008. PMid:18234075 PMCid:PMC2241838. http://dx.doi.org/10.1186/1741-7015-6-1

TRONCHIN, D. M. R; TSUNECHIRO, M. A. Prematuros de muito baixo peso: do nascimento ao primeiro ano de vida. Revista Gaúcha de Enfermagem, Porto Alegre, v. 28, n. 1, p. 79-88, 2007.

VENÂNCIO, S. I; ALMEIDA, H. Método Mãe Canguru: aplicação no Brasil, evidências científicas e impacto sobre o aleitamento materno. Jornal de Pediatria, São Paulo, v. 80 , p. S173-180, 2004. Suplemento 5.

WORLD HEALTH ORGANIZATION - WHO. Recommended definitions, terminology and format for statistical tables related to the perinatal period and use of a new certificate for cause of perinatal deaths. Modifications recommended by FIGO as amended October 14, 1976. Acta Obstet Gynecol Scand, Malden, v. 56, n. 3, p. $247-53,1977$.

\section{Contribuição dos Autores}

Mariana de Paiva Franco e Cristiane Paiva Alves participaram da coleta e organização dos dados, da organização de fontes, redação, análise e revisão do texto. 